\title{
BMJ Open Protocol for the PREHAB study- Pre-operative Rehabilitation for reduction of Hospitalization After coronary Bypass and valvular surgery: a randomised controlled trial
}

Andrew N Stammers, ${ }^{1,2}$ D Scott Kehler, ${ }^{1,2}$ Jonathan Afilalo, ${ }^{3}$ Lorraine J Avery, ${ }^{4}$ Sean M Bagshaw, ${ }^{5}$ Hilary P Grocott, ${ }^{6,7}$ Jean-Francois Légaré, ${ }^{8}$ Sarvesh Logsetty, ${ }^{6}$ Colleen Metge, ${ }^{4,9}$ Thang Nguyen, ${ }^{10}$ Kenneth Rockwood, ${ }^{11}$ Jitender Sareen, ${ }^{9,12}$ Jo-Ann Sawatzky, ${ }^{2,13}$ Navdeep Tangri, ${ }^{14}$ Nicholas Giacomantonio, ${ }^{15}$ Ansar Hassan, ${ }^{16}$ Todd A Duhamel, ${ }^{1,2,17}$ Rakesh C Arora ${ }^{2,6}$

To cite: Stammers AN, Kehler DS, Afilalo J, et al. Protocol for the PREHAB study-Pre-operative Rehabilitation for reduction of Hospitalization After coronary Bypass and valvular surgery: a randomised controlled trial. BMJ Open 2015;5:e007250. doi:10.1136/bmjopen-2014007250

- Prepublication history for this paper is available online. To view these files please visit the journal online (http://dx.doi.org/10.1136/ bmjopen-2014-007250).

Received 19 November 2014 Accepted 27 November 2014

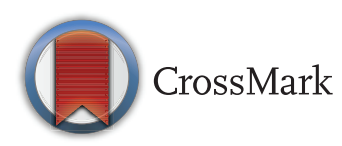

For numbered affiliations see end of article.

Correspondence to Dr Rakesh C Arora; rarora@sbgh.mb.ca

\section{ABSTRACT}

Introduction: Frailty is a geriatric syndrome characterised by reductions in muscle mass, strength, endurance and activity level. The frailty syndrome, prevalent in $25-50 \%$ of patients undergoing cardiac surgery, is associated with increased rates of mortality and major morbidity as well as function decline postoperatively. This trial will compare a preoperative, interdisciplinary exercise and health promotion intervention to current standard of care (StanC) for elective coronary artery bypass and valvular surgery patients for the purpose of determining if the intervention improves 3-month and 12-month clinical outcomes among a population of frail patients waiting for elective cardiac surgery.

Methods and analysis: This is a multicentre, randomised, open end point, controlled trial using assessor blinding and intent-to-treat analysis.

Two-hundred and forty-four elective cardiac surgical patients will be recruited and randomised to receive either StanC or StanC plus an 8-week exercise and education intervention at a certified medical fitness facility. Patients will attend two weekly sessions and aerobic exercise will be prescribed at $40-60 \%$ of heart rate reserve. Data collection will occur at baseline, 1-2 weeks preoperatively, and at 3 and 12 months postoperatively. The primary outcome of the trial will be the proportion of patients requiring a hospital length of stay greater than 7 days.

Potential impact of study: The healthcare team is faced with an increasingly complex older adult patient population. As such, this trial aims to provide novel evidence supporting a health intervention to ensure that frail, older adult patients thrive after undergoing cardiac surgery.

Ethics and dissemination: Trial results will be published in peer-reviewed journals, and presented at national and international scientific meetings. The University of Manitoba Health Research Ethics Board

\section{Strengths and limitations of this study}

- Multicentre, randomised trial.

- Does not capture urgent or emergent patients with critical cardiac illness.

- Does not capture the very frail older patient.

has approved the study protocol V.1.3, dated 11 August 2014 (H2014:208).

Trial registration number: The trial has been registered on ClinicalTrials.gov, a registry and results database of privately and publicly funded clinical studies (NCT02219815).

\section{INTRODUCTION}

Owing to an ageing demographic, older and increasingly frail patients are now being referred for cardiac surgery. In fact, the proportion of patients aged 75 years and older undergoing cardiac procedures increased from $16 \%$ in 1990 to up to $25 \%$ in most recent estimates. ${ }^{1}$ The surgical process in this population is complicated by the multiple comorbidities in addition to the underlying cardiac dysfunction presented by these frail patients. Previous studies have demonstrated that frail patients, typically with higher levels of concurrent medical comorbidities, often experience higher rates of postoperative morbidity, mortality and prolonged hospital length of stay (LOS) with associated increased burden on the healthcare system. ${ }^{2-5}$

Frailty is a geriatric syndrome, defined as an increased vulnerability to stressors leading 
to a state of decreased physiological resistance. ${ }^{6}{ }^{7}$ It is characterised by a "constellation of symptoms and signs [that] describe the heterogeneous response of older adults to physiological and metabolic challenges." While frailty is not necessarily synonymous with chronological age, it is more prevalent among the older adult population and is associated with up to a threefold increased risk of mortality or major morbidity postsurgery. ${ }^{9}$ A recent systematic review identified that frailty had a strong positive relationship with major adverse cardiac and cerebrovascular events in patients undergoing cardiac surgery $(\mathrm{OR}=4.89) .{ }^{10}$ Thus, it has become critically important for healthcare systems to develop strategies designed to improve clinical outcomes in this high-risk population undergoing cardiac surgery.

Since the phenotype of frailty is characterised by reductions in muscle mass, strength, endurance and activity level, ${ }^{7}$ cardiac rehabilitation (CR) is ideally suited to counteract these impairments and improve an individual's frailty status. A recent review of randomised trials concluded that multidisciplinary programmes were a promising therapeutic strategy for frail, older adults, although none of these specifically targeted these patients preoperatively. ${ }^{11}$ Thus, there is a strong biological and epidemiological rationale to investigate CR in this setting.

In Canada, when elective patients require cardiac surgery, they are placed on a waiting list for a period ranging from 1 to 4 months. While the wait period for elective patients is generally considered safe, it has been documented that patients on surgical waiting lists engage in very little physical activity as they wait for their procedure. ${ }^{12-14}$ A lack of physical activity can be problematic in an already deconditioned, frail cohort of patients because, at present, the current standard of care (StanC) does not provide a formal process to engage patients in physical activity during the waiting period. Therefore, the period of time prior to elective cardiac surgery presents a significant opportunity to optimise preoperative risk factors particularly in vulnerable patients, such as the frail, older adult population. The present study aims to reduce frailty in the high-risk, older adult patient to improve postsurgical outcomes of elective cardiac surgery.

CR has been shown to decrease morbidity and mortality in patients with established and unrepaired cardiac disease, ${ }^{14-17}$ and to be safe for patients who are elderly $^{18-22}$ and suffer heart failure, ${ }^{1623-25}$ in both hospital and community-based settings. ${ }^{15} 26 \quad 27 \quad \mathrm{~A}$ meta-analysis of randomised controlled trials reported that patients attending CR have a $20 \%$ and $26 \%$ relative reduction in all-cause morbidity and cardiac mortality, respectively, as compared with patients not attending CR. ${ }^{28}$ The proposed study has been designed according to the evidence-based, best practice guidelines published by the Canadian Association of Cardiac Rehabilitation, ${ }^{29}$ the recently published Canadian Cardiovascular Society Quality Indicators for $\mathrm{CR}^{30}$ and the guidelines for exercise prescription in patients with cardiovascular disease according to the American College of Sports Medicine. ${ }^{31}$

Although the benefits of postsurgical CR are well documented, less is known about the role that preoperative exercise may have in the trajectory of a patient undergoing heart surgery. A recent systematic review reported on the benefits of preoperative physical therapy in preventing postoperative pulmonary complications in patients undergoing cardiac surgery. ${ }^{32}$ Eight randomised controlled trials were included in the review, which found that preoperative physical therapy was effective in reducing postoperative pulmonary complications and length of hospital stay. However, the majority of the interventions used inspiratory muscle training and breathing exercises preoperatively and thus, did not address the potential benefit of exercise and education interventions in this population. In a seminal study by Arthur et $a l^{33} 249$ low-risk patients awaiting elective coronary artery bypass graft (CABG) surgery were randomised to an 8-week preoperative CR programme or to the StanC. Patients in the intervention group completed two exercise sessions per week and attended additional educational sessions on risk factor modifications. Following surgery, these patients experienced shorter intensive care unit (ICU) and hospital LOS postoperatively and an improved quality of life compared with controls. ${ }^{33}$ Notably, these promising results persisted for 6 months following surgery, indicating the potential long-term benefit of preoperative risk factor reduction. However, this particular study was conducted in relatively low-risk patients and thus, did not address the unique needs of frail, older adult patients that are now commonly being referred for cardiac surgery.

In a pilot, randomised control trial, ${ }^{12} 17$ preoperative elective CABG surgery patients were randomised to StanC or to an exercise and health education intervention. The intervention involved a 2-day weekly programme consisting of exercise and education classes for $60 \mathrm{~min}$ per session. Walking distance was evaluated using a 6 min walk test $(6 \mathrm{MWT})$, while gait speed was evaluated using the $5 \mathrm{~m}$ gait speed test. Walking distance remained unchanged in the StanC group; however, the intervention group increased their walking distance by 132 and $145 \mathrm{~m}$ at the preoperative and 3 months postoperative assessments, respectively. Gait speed was improved in the intervention group, by $27 \%$ and $33 \%$ preoperatively and 3 months postoperatively, respectively, while remaining unchanged in the control group. This pilot, randomised controlled trial demonstrated the feasibility of implementing a preoperative exercise protocol to improve the patient's functional capacity postsurgery; however, this study was performed for a relatively small number of younger patients and was designed as a simple feasibility and safety trial.

The Pre-operative Rehabilitation for Reduction of Hospitalization After Coronary Bypass and Valvular Surgery (PREHAB) study is a randomised control trial 
comparing a preoperative, interdisciplinary, exercise intervention and cardiovascular health promotion intervention to current StanC for the purpose of determining if the programme improves 3-month and 12-month clinical outcomes among a population of frail patients waiting for elective cardiac surgery. The primary objectives of the trial are:

1. To determine if the PREHAB intervention reduces the proportion of frail older adult patients requiring a prolonged hospital LOS greater than 7 days.

2. To determine if the PREHAB intervention influences frailty, exercise capacity, physical activity behaviour, in-hospital complications and health-related quality of life (HRQoL).

We hypothesise that the performance of an interdisciplinary programme preoperatively in patients awaiting elective cardiac surgery will reduce the proportion of frail, older adult patients requiring a postoperative hospital LOS greater than 7 days. We also hypothesise that the intervention will: (1) reduce frailty; (2) improve exercise capacity; (3) improve self-managed physical activity behaviour; (4) improve in-hospital outcomes; (5) improve clinical outcomes 3 months and 1 year postoperatively; and (6) improve HRQoL.

\section{METHODS AND ANALYSIS}

The PREHAB study is a prospective, randomised, open, blinded end point (PROBE) ${ }^{34}$ controlled trial using assessor blinding and intention-to-treat analysis. To date, no high-quality study has prospectively examined the impact of preoperative CR in the frail-older adult population undergoing cardiac surgery. Thus, the PREHAB trial has been designed to address a noticeable knowledge gap in the current literature. The trial has been registered on National Institutes of Health ClinicalTrials. gov (NCT02219815). The study is funded by an Operating Grant from the Canadian Institutes of Health Research (326290), which was awarded following an independent peer-review process and supported by Technology Evaluation in the Elderly (TVN). We have used the Standard Protocol Items: Recommendations for Intervention Trials (SPIRIT) guidelines in reporting this clinical trial. ${ }^{35}$

\section{Setting}

The PREHAB trial will be conducted in three centres across Canada: (1) Winnipeg, Manitoba; (2) Halifax, Nova Scotia and (3) Saint John, New Brunswick. The study will recruit a total of 244 patients or 122 per study arm. Surgical procedures in study participants will be conducted at two academic, tertiary care hospitals (Winnipeg, Manitoba and Halifax, Nova Scotia, Canada) and one non-academic hospital (Saint John, New Brunswick, Canada) that perform cardiac surgery. These study sites were strategically chosen based on similar patient demographics and comparable surgical waiting list time. ${ }^{36}{ }^{37}$ Additionally, each of these hospitals are partnered with one or more community-based CR centres, which are certified medical fitness facilities dedicated to improving the health of the community through health promotion, disease prevention and rehabilitation services. These facilities offer expert guidance from certified professionals, innovative health enhancement programmes, and provide integrated medical, rehabilitative and fitness services. The CR centres will provide the infrastructure and programme expertise necessary for the implementation and delivery of the PREHAB intervention.

\section{Participant selection}

Inclusion criteria

1. Patients, aged 65 years or older, undergoing elective isolated CABG, aortic valve repair/replacement for moderate aortic stenosis or severe regurgitation, mitral valve repair/replacement for moderate stenosis or severe regurgitation, or combined/valve procedures.

2. Patients with Clinical Frailty Score (CFS) $\geq 4$ (vulnerable) and $<7$ (8=very severely frail, approaching end-of-life or $9=$ terminally ill) at time of acceptance for cardiac surgery.

3. Patients with an estimated $\geq 6$ week surgical waiting list time.

\section{Exclusion criteria}

1. Patients who have unstable or recent unstable cardiac syndrome as defined by:

A. Severe heart failure (New York Heart Association Class IV) or angina (Canadian Cardiovascular Society Class IV) symptoms.

B. Critical left main coronary disease.

C. Hospitalisation for arrhythmias, congestive heart failure or acute coronary syndrome prior to randomisation.

2. Patients who have severe left ventricular (LV) obstructive disease as defined by:

A. Severe aortic or mitral stenosis (aortic or mitral valve area $<1.0 \mathrm{~cm}^{2}$ or mean gradient $>40$ or $>10 \mathrm{~mm} \mathrm{Hg}$, respectively).

B. Dynamic LV outflow obstruction.

3. Patients who have demonstrated exercise-induced ventricular arrhythmias or have experienced a recent hospitalisation for arrhythmias.

4. Patients who have cognitive deficits that would preclude rehabilitation.

5. Patients who have physical limitations that would preclude rehabilitation.

6. Patients who are unable to attend the PREHAB programme.

\section{Screening}

Our strategy for rapid screening of frailty in the large numbers of incoming patients will utilise the nine-point CFS. ${ }^{38}$ We have defined a CFS of greater than or equal to 4 (classified as 'vulnerable') as an initial indicator of frailty. Following training, clinicians will complete a CFS 
for every new cardiac surgery consult at each of the participating sites. Patients with a CFS of 4-6 will be eligible for enrolment and notification will be sent to the local site coordinator (see figure 1 for patient flow). We will exclude severely frail patients with a CFS greater than or equal to 7 because of the physical limitations in these patients. Furthermore, we anticipate that this criterion will exclude less than $1 \%$ of the patients on the elective cardiac surgery wait list.

A medical director at each site will oversee all cases, and ensure patient eligibility and safety for programme initiation and throughout the duration of the study. All eligible and accepted patients will be evaluated at baseline for safety parameters and to ensure angina stability. Patients who are deemed appropriate for enrolment into the study will be randomised, following completion of the baseline data collection and confirmation of eligibility, to one of two trial arms-control (StanC) or treatment (PREHAB). All patients will undergo a baseline exercise stress test supervised by a cardiologist to ensure patient safety. Safety data, including new hospitalisation, worsening angina or heart failure and arrhythmias will be captured and all adverse events will be reportable to an independent data safety monitoring board (DSMB).

\section{Instrumentation}

Preoperative patient demographics, cardiac risk profile, procedure data, surgical risk scores (EuroSCORE II and STS-PROMM) ${ }^{39-41}$ medication profile, cardiac risk factors and cardiac illness severity are routinely collected for all patients in pre-existing site surgical databases. Each site will also collect covariates of comorbidities using the Charleston Co-morbidity Index, Functional Co-morbidity Index, ${ }^{42}$ Older Americans Resources and Services scale for activities of daily living (ADL) and instrumental $\mathrm{ADL},{ }^{43}$ as well as postoperative complications and postoperative hospital LOS. Cognitive function will be measured using the Montreal Cognitive Assessment (MoCA). ${ }^{44} 45$ The Patient Health Questionnaire-9 (PHQ-9) will assess depressive

Figure 1 Patient flow (CFS, Clinical Frailty Score; 6MWT, 6 min walk test; SPPB, Short Performance Physical Battery Test; PREHAB, Pre-operative Rehabilitation for Reduction of Hospitalization After Coronary Bypass and Valvular Surgery).

\section{Patients aged 65+ with a CFS 4-6 scheduled for elective cardiac surgery}

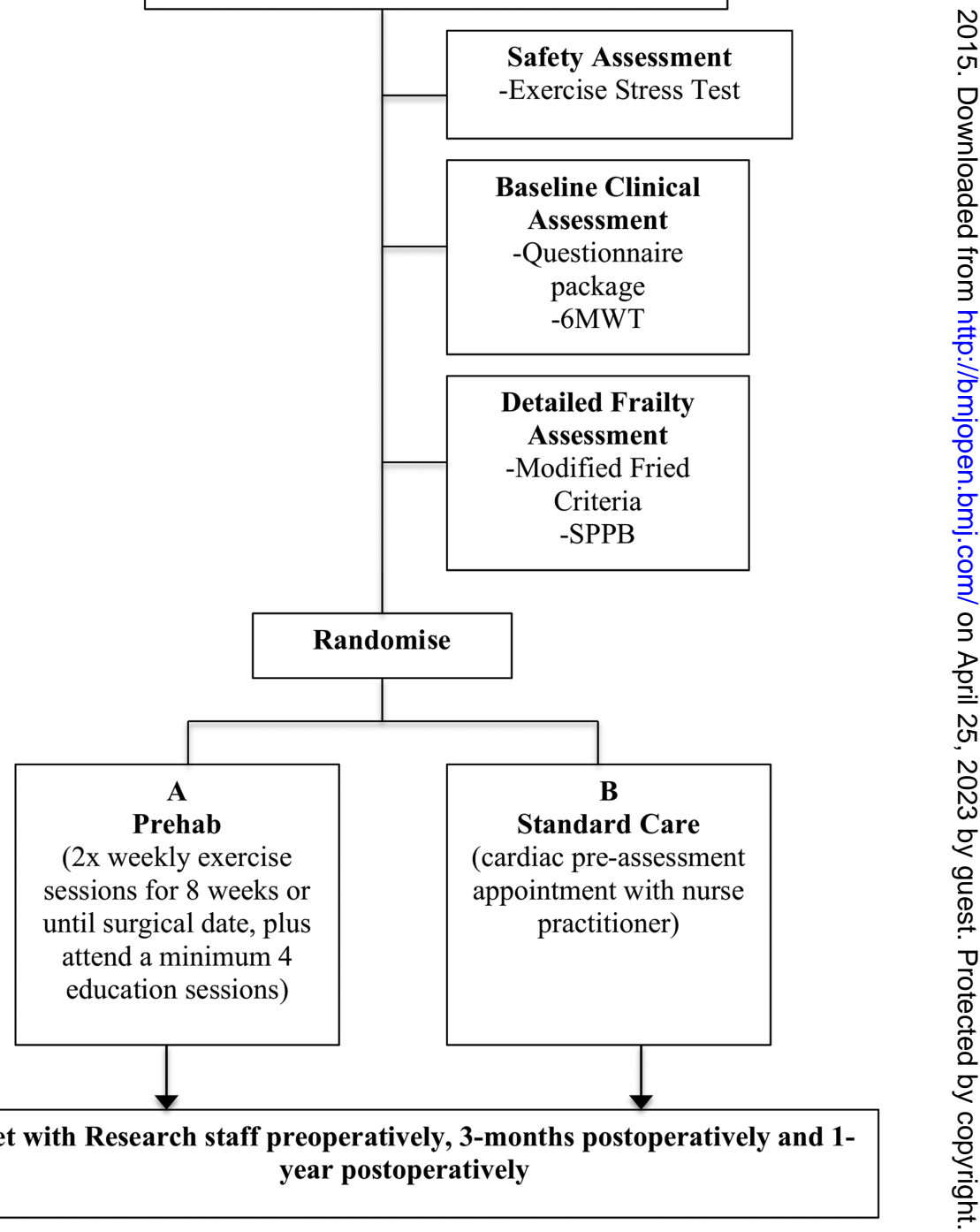


symptoms ${ }^{46}$ while the Generalized Anxiety Disorder Scale 7-Item (GAD-7) will be used to assess generalised anxiety disorder. ${ }^{47}$ The Short Form-12 (SF-12 V.2) and the EuroQual-5D (EQ-5D) will be administered as validated measures of HRQoL. ${ }^{48} 49$ Also included in the data collection package are cardiac-specific questionnaires-the Seattle Angina Questionnaire (SAQ), Kansas City Cardiomyopathy Questionnaire (KCCQ) and the International Physical Activity Questionnaire (IPAQ). Objectively measured physical activity will be quantified using multidirectional accelerometry (Actical Physical Activity Monitors) for a period of 7 days at each data collection time point. This technique is considered the gold standard for assessing daily physical activity accumulation. $^{50} 51$ Patients will also undergo a standard $6 \mathrm{MWT}^{52-56}$ and will repeat the 6MWT at the preoperative time point in both the control and PREHAB groups to determine the impact of the intervention on physical capacity. The 6MWT was chosen because it requires minimal infrastructure and personnel to complete, has been demonstrated to be a valid prognostic tool for patient outcomes among cardiac surgery patients, ${ }^{12} 57$ and is comparable to an exercise stress test. ${ }^{52-56}$ The Modified Fried Criteria ${ }^{6} 758$ will be used to assess changes in frailty, in addition to secondary measures of frailty employing the Short Performance Physical Battery Test (SPPB). ${ }^{59}$

\section{Assessor blinding}

Individuals performing the assessments preoperatively, 3 months postoperatively and 1 year postoperatively will be blinded to the study arm in which the patient was assigned. Additionally, hospital staff, including nurses and surgeons, will be blinded to the study arm in which the patient has been assigned.

\section{Randomisation}

Randomisation (with concealed allocation) will be stratified by study location and will occur on a 1:1 basis in permuted blocks.

\section{Standard care}

Currently, patients awaiting cardiac surgery receive StanC (see table 1) where they are at present given no specific, routine instruction while awaiting their procedure.

\section{Intervention}

Patients randomised to the intervention group will receive, in addition to the above StanC, an 8-week comprehensive exercise therapy and education programme at a CR facility (see table 1 ). This programme will target both the physical, and psychological and social cognitive aspects of cardiac disease and frailty. In brief, participants will be required to complete an intake health status assessment by the CR team, including a physiotherapist, cardiovascular nurse and dietitian, and complete a symptom-limited, graded exercise stress test according to the American College of Sports Medicine Guidelines for Exercise Testing and Prescription. ${ }^{31}$ This assessment will provide the basis for exercise prescription in our population, which will include an individualised, symptom-limited exercise programme. Intervention group patients will be required to complete at least two sessions of supervised, structured exercise sessions per week for a total period of 8 weeks. Attendance will be monitored by the local CR site. Participants will complete a warm-up programme of approximately $15 \mathrm{~min}$ at the beginning of each session. Subsequently, aerobic exercise will be prescribed at $40-60 \%$ of heart rate reserve (Karvonen Formula) based

\section{Table 1 Comparison of PREHAB and standard care}

\begin{tabular}{|c|c|}
\hline Standard care & PREHAB intervention \\
\hline $\begin{array}{l}\text { Patient advised to rest and participate in light-intensity } \\
\text { physical activity } \\
1-2 \text { weeks prior to the scheduled surgical date, patient } \\
\text { attends single, } 3 \mathrm{~h} \text { cardiac assessment with nurse } \\
\text { practitioner and cardiac anaesthesiologist } \\
\text { Patient receives counselling on basic healthy living } \\
\text { behaviours }\end{array}$ & $\begin{array}{l}\text { Patient will receive, in addition to standard care, } 8 \text {-week } \\
\text { exercise and education programme at community-based } \\
\text { cardiac rehabilitation facility } \\
\text { Patient will undergo an intake health status assessment } \\
\text { Patient will be required to attend two supervised exercise } \\
\text { sessions per week: } \\
\text { 1. Warm-up of approximately } 15 \text { min including stretching } \\
\text { 2. } 10-30 \text { min of aerobic exercise at } 40-60 \% \text { of heart rate } \\
\text { reserve progressing to high-intensity interval training up to } \\
85 \% \text { of aerobic capacity, based on recommendations by } \\
\text { cardiologist } \\
\text { 3. } 10 \text { min cool-down period } \\
\text { Patient will participate in four educational sessions covering } \\
\text { a range of topics including risk factor reduction, medication } \\
\text { use, cardiovascular physiology, smoking cessation, healthy } \\
\text { eating, stress management and promotion of self-managed } \\
\text { care }\end{array}$ \\
\hline
\end{tabular}

PREHAB, Pre-operative Rehabilitation for Reduction of Hospitalization After Coronary Bypass and Valvular Surgery. 
on baseline exercise stress test data. ${ }^{60}$ Aerobic exercise will be approximately 10-30 min in duration, depending on individual tolerance and current level of conditioning. Aerobic exercise prescription will progress to highintensity exercise in the context of symptom-limited, interval training up to $85 \%$ of maximal aerobic capacity based on recommendations by a cardiologist and medical fitness facility staff. ${ }^{29}$ All individual sessions will be concluded with a 10 min cool down period. In addition to the prescribed exercise programme, patients will be required to participate in four educational sessions tailored towards self-management for CR and healthy living practices. These education sessions will be directly relevant to the patient population and will cover a range of topics, including risk factor reduction, medication use, cardiovascular physiology, smoking cessation, healthy eating, stress management and promotion of self-managed care. Principles of shared decision-making will be utilised with the PREHAB intervention, where training for providers and patients involved in the intervention will have shared control of treatment decisions. ${ }^{61-63}$

\section{Data collection and management}

All participants will meet with the research staff at five time points: (1-2) twice at time of enrolment after the patient is referred for a surgical procedure (ie, baseline preintervention); (3) 1 week preoperatively; (4) 3 months postoperatively and (5) 1 year postoperatively. Data collection will occur from both written and electronic medical record sources (ie, patient information systems, where applicable). Existing perioperative surgical, ICU and hospital data will be used to capture patient demographics, procedure urgency, intraoperative procedure and anaesthetic variables, cardiopulmonary bypass information, blood product utilisation, mechanical ventilation, delirium/coma, ICU and hospital LOS, major adverse events, infection and ICU, and hospital recidivism are collected in institutional databases.

Local research staff will enter de-identified study data onto a trial-specific electronic case report form through REDCap, a secure, web-based application for managing online surveys and databases. ${ }^{64}$ The data will be housed on a secure server located at the University of Manitoba Faculty of Medicine.

\section{Statistical analysis}

The sample size calculation was based on the primary objective of the study-hospital LOS. Preliminary data collected by our group demonstrated the proportion of frail patients with a hospital LOS greater than 7 days to be $58.3 \%$. For the intervention group, based on improvements in functional capacity in our pilot trial ${ }^{12}$ and previous work by Arthur et $a l^{33}$ we believe an absolute $20 \%$ reduction in the proportion of patients that require prolonged hospital LOS greater than 7 days is feasible. A sample size of 194 individuals (97 per study arm) will be required for a two-tailed test at an $\alpha$ of 0.05 and power of $80 \%$. We are estimating a $20 \%$ dropout rate given the target population of the study and thus, plan to recruit 244 patients (122 in each study arm) to achieve an eventual sample size of 194 after dropout.

Objective 1: To determine if PREHAB reduces the proportion of frail older adult patients requiring a prolonged hospital LOS of greater than 7 days. A univariable analysis will be undertaken to determine if hospital LOS greater than 7 days is associated with frailty and other patient-level factors. A stepwise multivariable logistic regression model will then be developed to determine if PREHAB attendance is independently associated with a decreased risk of prolonged hospital LOS greater than 7 days. As a secondary analysis, hospital LOS will also be compared as a continuous variable between PREHAB and the StanC groups using a Mann-Whitney test. A multivariable model for hospital LOS will also be developed utilising generalised linear models with Poisson or Negative Binomial distribution.

Objective 2: Does attending PREHAB influence frailty, exercise capacity, physical activity behaviour, in-hospital complications, and HRQoL? The Modified Fried Criteria will be the primary frailty outcome over time from baseline to preoperative or from baseline to the long-term follow-up assessments. The primary measure of exercise capacity will be the 6MWT and will be analysed with univariable linear regression models to evaluate the association between the 6MWT and PREHAB attendance. Accelerometers will be used to objectively measure the number of minutes spent performing moderate to vigorous physical activity and the total physical activity per day for a period of 7 days and compared between groups. A composite outcome for a major adverse cardiac event (ie, in hospital stroke, myocardial infarction, renal failure requiring dialysis and death) and delirium will be compared between groups using a $\chi^{2}$ or Fisher's exact test. A multivariable logistic regression will be used to identify if PREHAB is independently associated with in-hospital delirium. The SF-12V.2 and EQ-5D scores, after normative standardisation, will be analysed through use of a repeated measures analysis of variance to allow for a standardised group and group/ time interaction effect estimation.

\section{ETHICS}

Ethics approval will be obtained at each individual study site. Any substantial protocol amendments will be sent to the local ethics committee for approval as per standard regulatory requirements at the institution. All study sites will then be informed of the amendments.

\section{Informed consent}

During an initial meeting with a cardiac surgeon, eligible and interested patients will be approached by a research assistant (RA) who will provide the patient with further details of the study. The patient will be informed about the trial by the RA and provided with a copy of 
the patient information and consent form. Patients will be provided with adequate amount of time to consider their participation in the trial and will be given an opportunity to ask questions. If the patient decides to participate in the study, they will be asked to provide written consent, which will then be countersigned by the RA. All participants are free to withdraw from the study at any time, without any prejudice to future medical treatment.

\section{End of trial}

The trial will end when 244 patients have been enrolled and the final patient has completed their 1-year follow-up appointment.

\section{Trial monitoring and oversight}

The study management and steering committee is responsible for ensuring that the study meets the proposed milestones and deadlines. They will also be responsible for all aspects of the study design, management, ethical conduct, analysis and dissemination of results.

Safety data, including new hospitalisation, worsening angina or heart failure and arrhythmias, will be captured and all adverse events will be reportable to the DSMB. The DSMB is an independent group of experts that advises study investigators. They are responsible to periodically evaluate the study data for participant safety and study conduct, in addition to making recommendations concerning the modification and/or termination of the trial.

\section{DISSEMINATION}

Results will be presented at scientific meetings and published in peer-reviewed journals. All publications and presentations related to the study will be authorised and reviewed by the study investigators. Authorship will be determined based on internationally agreed on criteria and journal guidelines for authorship. A major component of the proposed project is to collect information that is appropriate to enable knowledge users to obtain the information they need to inform new initiatives within their own organisations. The integrated knowledge translation (iKT) approach enhances the likelihood that the PREHAB intervention will be implemented in the Canadian healthcare system and be sustainable over the long term. By using an iKT approach and best practice evidence to inform a model of care for frail elderly persons requiring cardiac surgery, it is anticipated that the project will influence the healthcare system in a variety of ways. The study protocol also includes a process for sharing study outcomes with participants. Specifically, study participants will have the option at the time of consent to indicate their interest in receiving a summary of study findings. This summary will then be sent to participants on completion of the study.

\section{Trial status}

The study is not yet recruiting patients. The first patient will be recruited in January 2015, and we expect recruitment to be complete in March of 2016. The expected completion date of the project, including all 1-year follow-up appointments, is May 2017.

\section{Author affiliations}

${ }^{1}$ Faculty of Kinesiology \& Recreation Management, Health, Leisure \& Human Performance Research Institute, University of Manitoba, Winnipeg, Manitoba, Canada

${ }^{2}$ Institute of Cardiovascular Sciences, St. Boniface Hospital Research Centre, Winnipeg, Manitoba, Canada

${ }^{3}$ Divisions of Cardiology and Clinical Epidemiology, Department of Medicine, Jewish General Hospital, McGill University, Montreal, Quebec, Canada

${ }^{4}$ Winnipeg Regional Health Authority Cardiac Sciences Program, Winnipeg, Manitoba, Canada

${ }^{5}$ Division of Critical Care Medicine, Faculty of Medicine and Dentistry, University of Alberta, Edmonton, Alberta, Canada

${ }^{6}$ Department of Surgery, Faculty of Medicine, University of Manitoba, Winnipeg, Manitoba, Canada

${ }^{7}$ Department of Anesthesia \& Perioperative Medicine, Faculty of Medicine, University of Manitoba, Winnipeg, Manitoba, Canada

${ }^{8}$ Division of Cardiac Surgery, Department of Surgery, Dalhousie University, Halifax, Nova Scotia, Canada

${ }^{9}$ Department of Community Health Sciences, Faculty of Medicine, University of Manitoba, Winnipeg, Manitoba, Canada

${ }^{10}$ Section of Cardiology, Faculty of Medicine, University of Manitoba,

Winnipeg, Manitoba, Canada

${ }^{11}$ Division of Geriatric Medicine, Department of Medicine, Dalhousie

University, Halifax, Nova Scotia, Canada

${ }^{12}$ Department of Psychiatry, Faculty of Medicine, University of Manitoba, Winnipeg, Manitoba, Canada

${ }^{13}$ Faculty of Health Sciences, College of Nursing, University of Manitoba, Winnipeg, Manitoba, Canada

${ }^{14}$ Section of Nephrology, Faculty of Medicine, University of Manitoba,

Winnipeg, Manitoba, Canada

${ }^{15}$ Division of Cardiology, Department of Medicine, Dalhousie University, Halifax, Nova Scotia, Canada

${ }^{16}$ Department of Cardiac Surgery, New Brunswick Heart Centre, Saint John Regional Hospital, Saint John, New Brunswick, Canada

${ }^{17}$ Department of Physiology, University of Manitoba, Winnipeg, Manitoba, Canada

Acknowledgements The authors would like to acknowledge the infrastructure support provided by the St. Boniface Hospital Research Centre, and the Health Leisure and Human Performance Research Institute at the University of Manitoba.

Contributors JA, LJA, SMB, HPG, J-FL, SL, CM, TN, KR, JS, J-AS, NT, NG, $A H, T A D$ and RCA were involved in conception and design of research. NG, $A H, T A D$ and RCA co-lead investigators. ANS, DSK, TAD and RCA obtained ethics approval. ANS and DSK prepared figures. ANS, DSK, TAD and RCA drafted manuscript. ANS, DSK, JA, HPG, SL, J-AS, NT, TAD and RCA edited and revised the manuscript. ANS, DSK, JA, LJA, SMB, HPG, J-FL, SL, CM, TN, KR, JS, J-AS, NT, NG, AH, TAD and RCA approved the final version of the manuscript.

Funding The work was supported by an Operating Grant from the Canadian Institutes of Health Research, grant number 326290. It is also supported by Technology Evaluation in the Elderly (TVN). TAD was supported by a Manitoba Health Research Council (MHRC) Establishment Grant. ANS was supported by a CIHR Canada Graduate Scholarship. DSK was supported by an MHRC Studentship, a CIHR Strategic Training Initiative in Health Research (STIHR) Knowledge Translation Canada Student Fellowship, a CIHR STIHR Population Intervention for Chronic Disease Prevention Fellowship, and the Heart and Stroke Foundation Dr Dexter Harvey Award.

Competing interests None. 
Ethics approval The trial protocol V.1.3 and participant information sheets have been reviewed by the University of Manitoba Health Research Ethics Board and have been approved (H2014:208).

Provenance and peer review Not commissioned; peer reviewed for ethical and funding approval prior to submission.

Open Access This is an Open Access article distributed in accordance with the Creative Commons Attribution Non Commercial (CC BY-NC 4.0) license, which permits others to distribute, remix, adapt, build upon this work noncommercially, and license their derivative works on different terms, provided the original work is properly cited and the use is non-commercial. See: http:// creativecommons.org/licenses/by-nc/4.0/

\section{REFERENCES}

1. Afilalo J, Mottillo S, Eisenberg MJ, et al. Addition of frailty and disability to cardiac surgery risk scores identifies elderly patients at high risk of mortality or major morbidity. Circ Cardiovasc Qual Outcomes 2012;5:222-8.

2. Green $\mathrm{P}$, Woglom $\mathrm{AE}$, Genereux $\mathrm{P}$, et al. The impact of frailty status on survival after transcatheter aortic valve replacement in older adults with severe aortic stenosis: a single-center experience. JACC Cardiovasc Interv 2012;5:974-81.

3. Lee DH, Buth KJ, Martin B-J, et al. Frail patients are at increased risk for mortality and prolonged institutional care after cardiac surgery. Circulation 2010;121:973-8.

4. Singh M, Rihal CS, Lennon RJ, et al. Influence of frailty and health status on outcomes in patients with coronary disease undergoing percutaneous revascularization. Circ Cardiovasc Qual Outcomes 2011:4:496-502.

5. Sündermann S, Dademasch A, Rastan A, et al. One-year follow-up of patients undergoing elective cardiac surgery assessed with the Comprehensive Assessment of Frailty test and its simplified form. Interact Cardiovasc Thorac Surg 2011:13:119-23; discussion 123.

6. Fried LP, Ferrucci L, Darer J, et al. Untangling the concepts of disability, frailty, and comorbidity: implications for improved targeting and care. J Gerontol A Biol Sci Med Sci 2004;59:255-63.

7. Fried LP, Tangen CM, Walston J, et al. Frailty in older adults evidence for a phenotype. J Gerontol A Biol Sci Med Sci 2001:56:M146-57.

8. Quinlan N, Marcantonio ER, Inouye SK, et al. Vulnerability: the crossroads of frailty and delirium. J Am Geriatr Soc 2011;59(Suppl 2):S262-8.

9. Afilalo J, Eisenberg MJ, Morin J-F, et al. Gait speed as an incremental predictor of mortality and major morbidity in elderly patients undergoing cardiac surgery. J Am Coll Cardiol 2010;56:1668-76.

10. Sepehri A, Beggs T, Hassan A, et al. The impact of frailty on outcomes after cardiac surgery: a systematic review. J Thorac Cardiovasc Surg 2014;148:3110-17.

11. Bibas L, Levi M, Bendayan M, et al. Therapeutic interventions for frail elderly patients: part I. Published randomized trials. Prog Cardiovasc Dis 2014:57:134-43.

12. Sawatzky J-AV, Kehler DS, Ready AE, et al. Prehabilitation program for elective coronary artery bypass graft surgery patients: a pilot randomized controlled study. Clin Rehabil 2014;28:648-57.

13. Nery RM, Barbisan JN. Effect of leisure-time physical activity on the prognosis of coronary artery bypass graft surgery. Rev Bras Cir Cardiovasc 2010;25:73-8.

14. Mooney M, Fitzsimons D, Richardson G. 'No more couch-potato!' Patients' experiences of a pre-operative programme of cardiac rehabilitation for those awaiting coronary artery bypass surgery. Eur J Cardiovasc Nurs 2007;6:77-83.

15. Goel K, Lennon RJ, Tilbury RT, et al. Impact of cardiac rehabilitation on mortality and cardiovascular events after percutaneous coronary intervention in the community. Circulation 2011;123:2344-52.

16. Díaz-Buschmann I, Jaureguizar KV, Calero MJ, et al. Programming exercise intensity in patients on beta-blocker treatment: the importance of choosing an appropriate method. Eur J Prev Cardiol 2014;:21:1474-80.

17. Cote AT, Bredin SSD, Phillips AA, et al. Left ventricular mechanics and arterial-ventricular coupling following high-intensity interval exercise. J Appl Physiol 2013;115:1705-13.

18. Audelin MC, Savage PD, Ades PA. Exercise-based cardiac rehabilitation for very old patients ( $>=75$ years): focus on physical function. J Cardiopulm Rehabil Prev 2008;28:163-73.

19. Ades PA, Ballor DL, Ashikaga T, et al. Weight training improves walking endurance in healthy elderly persons. Ann Intern Med 1996;124:568-72.
20. Suaya JA, Stason WB, Ades PA, et al. Cardiac rehabilitation and survival in older coronary patients. J Am Coll Cardiol 2009;54:25-33.

21. Rengo G, Galasso G, Vitale DF, et al. An active lifestyle prior to coronary surgery is associated with improved survival in elderly patients. J Gerontol A Biol Sci Med Sci 2010;65:758-63.

22. Busch JC, Lillou D, Wittig G, et al. Resistance and balance training improves functional capacity in very old participants attending cardiac rehabilitation after coronary bypass surgery. J Am Geriatr Soc 2012;60:2270-6.

23. Chrysohoou C, Tsitsinakis G, Vogiatzis I, et al. High intensity, interval exercise improves quality of life of patients with chronic heart failure: a randomized controlled trial. QJM 2014;107:25-32.

24. Zoll J, N'Guessan B, Ribera F, et al. Preserved response of mitochondrial function to short-term endurance training in skeletal muscle of heart transplant recipients. J Am Coll Cardiol 2003;42:126-32.

25. Nilsson BB, Westheim A, Risberg MA. Effects of group-based high-intensity aerobic interval training in patients with chronic heart failure. Am J Cardiol 2008;102:1361-5.

26. Scheinowitz M, Harpaz D. Safety of cardiac rehabilitation in a medically supervised, community-based program. Cardiology 2005;103:113-17

27. Aamot I-L, Forbord SH, Gustad K, et al. Home-based versus hospital-based high-intensity interval training in cardiac rehabilitation: a randomized study. Eur J Prev Cardiol 2014;21:1070-8.

28. Taylor RS, Brown A, Ebrahim S, et al. Exercise-based rehabilitation for patients with coronary heart disease: systematic review and meta-analysis of randomized controlled trials. Am J Med 2004:116:682-92

29. Stone JA, Arthur HM; Canadian Association of Cardiac Rehabilitation Guidelines Writing Group. Canadian guidelines for cardiac rehabilitation and cardiovascular disease prevention, second edition, 2004: executive summary. Can J Cardiol 2005;21(Suppl D):3D-19D.

30. Grace SL, Poirier P, Norris CM, et al. Pan-Canadian development of cardiac rehabilitation and secondary prevention quality indicators. Can J Cardiol 2014;30:945-8.

31. Thompson PD, Arena R, Riebe D, et al. ACSM's new preparticipation health screening recommendations from ACSM's guidelines for exercise testing and prescription, ninth edition. Curr Sports Med Rep 2013;12:215-17.

32. Hulzebos EHJ, Smit Y, Helders PPJM, et al. Preoperative physical therapy for elective cardiac surgery patients. Cochrane Database Syst Rev 2012;11:CD010118.

33. Arthur HM, Daniels C, McKelvie R, et al. Effect of a preoperative intervention on preoperative and postoperative outcomes in low-risk patients awaiting elective coronary artery bypass graft surgery. A randomized, controlled trial. Ann Intern Med 2000;133:253-62.

34. Hansson L, Hedner T, Dahlöf B. Prospective randomized open blinded end-point (PROBE) study. A novel design for intervention trials. Prospective Randomized Open Blinded End-Point. Blood Press 1992;1:113-19.

35. Chan A-W, Tetzlaff JM, Altman DG, et al. SPIRIT 2013 statement: defining standard protocol items for clinical trials. Ann Intern Med 2013;158:200-7.

36. Lamarche $\mathrm{Y}$, Sirounis D, Arora RC, et al. A survey of standardized management protocols after coronary artery bypass grafting surgery in Canadian intensive care units. Can J Cardiol 2011:27:705-10.

37. Martin B-J, Buth KJ, Arora RC, et al. Delirium as a predictor of sepsis in post-coronary artery bypass grafting patients: a retrospective cohort study. Crit Care 2010;14:R171.

38. Rockwood K, Song X, MacKnight C, et al. A global clinical measure of fitness and frailty in elderly people. CMAJ 2005;173:489-95.

39. Shahian DM, O'Brien SM, Filardo G, et al. The Society of Thoracic Surgeons 2008 cardiac surgery risk models: part 3-valve plus coronary artery bypass grafting surgery. Ann Thorac Surg 2009;88: S43-62.

40. Shahian DM, O'Brien SM, Filardo G, et al. The Society of Thoracic Surgeons 2008 cardiac surgery risk models: part 1-coronary artery bypass grafting surgery. Ann Thorac Surg 2009;88:S2-22.

41. O'Brien SM, Shahian DM, Filardo G, et al. The Society of Thoracic Surgeons 2008 cardiac surgery risk models: part 2-isolated valve surgery. Ann Thorac Surg 2009;88:S23-42.

42. Groll DL, To T, Bombardier C, et al. The development of a comorbidity index with physical function as the outcome. J Clin Epidemiol 2005;58:595-602.

43. Fillenbaum GG, Smyer MA. The development, validity, and reliability of the OARS multidimensional functional assessment questionnaire. $J$ Gerontol 1981;36:428-34.

44. Cameron J, Worrall-Carter L, Page K, et al. Screening for mild cognitive impairment in patients with heart failure: Montreal cognitive 
assessment versus mini mental state exam. Eur J Cardiovasc Nurs 2013;12:252-60.

45. Dong Y, Lee WY, Basri NA, et al. The Montreal Cognitive Assessment is superior to the Mini-Mental State Examination in detecting patients at higher risk of dementia. Int Psychogeriatr 2012;24:1749-55.

46. Kroenke K, Spitzer RL, Williams JB. The PHQ-9: validity of a brief depression severity measure. J Gen Intern Med 2001;16:606-13.

47. Herr NR, Williams JW, Benjamin S, et al. Does this patient have generalized anxiety or panic disorder? The Rational Clinical Examination systematic review. JAMA 2014;312:78-84.

48. Lacson $\mathrm{E}, \mathrm{Xu} \mathrm{J}$, Lin S-F, et al. A comparison of SF-36 and SF-12 composite scores and subsequent hospitalization and mortality risks in long-term dialysis patients. Clin J Am Soc Nephrol 2010;5:252-60.

49. Johnson JA, Coons SJ. Comparison of the EQ-5D and SF-12 in an adult US sample. Qual Life Res 1998;7:155-66.

50. TROST SG, MCIVER KL, PATE RR. Conducting accelerometer-based activity assessments in field-based research. [Miscellaneous Article]. Med Sci Sports Exerc 2005;37(11 Suppl): S531-43.

51. Colley RC, Garriguet D, Janssen I, et al. Physical activity of Canadian children and youth: accelerometer results from the 2007 to 2009 Canadian Health Measures Survey. Health Rep 2011;22:15-23.

52. Ross RM, Murthy JN, Wollak ID, et al. The six minute walk test accurately estimates mean peak oxygen uptake. BMC Pulm Med 2010;10:31.

53. Myers J, Prakash M, Froelicher V, et al. Exercise capacity and mortality among men referred for exercise testing. $N$ Engl J Med 2002;346:793-801.
54. Opasich C, De Feo S, Pinna GD, et al. Distance walked in the 6-minute test soon after cardiac surgery: toward an efficient use in the individual patient. Chest 2004;126:1796-801.

55. Beatty AL, Schiller NB, Whooley MA. Six-minute walk test as a prognostic tool in stable coronary heart disease: data from the heart and soul study. Arch Intern Med 2012;172:1096-102.

56. Enright PL. The six-minute walk test. Respir Care 2003;48:783-5.

57. Rasekaba T, Lee AL, Naughton MT, et al. The six-minute walk test: a useful metric for the cardiopulmonary patient. Intern Med $J$ 2009;39:495-501.

58. Bergman H, Ferrucci L, Guralnik J, et al. Frailty: an emerging research and clinical paradigm-issues and controversies. J Gerontol A Biol Sci Med Sci 2007;62:731-7.

59. Guralnik JM, Ferrucci L, Pieper CF, et al. Lower extremity function and subsequent disability consistency across studies, predictive models, and value of gait speed alone compared with the short physical performance battery. J Gerontol A Biol Sci Med Sci 2000;55:M221-31.

60. Karvonen J, Vuorimaa T. Heart rate and exercise intensity during sports activities. Practical application. Sports Med 1988;5:303-11.

61. Glasgow RE, Funnell MM, Bonomi AE, et al. Self-management aspects of the improving chronic illness care breakthrough series: implementation with diabetes and heart failure teams. Ann Behav Med 2002;24:80-7.

62. Glasgow RE, Nutting PA, King DK, et al. A practical randomized trial to improve diabetes care. J Gen Intern Med 2004;19:1167-74.

63. Charles C, Gafni A, Whelan T. Shared decision-making in the medical encounter: what does it mean? (or it takes at least two to tango). Soc Sci Med 1997;44:681-92.

64. Obeid JS, McGraw CA, Minor BL, et al. Procurement of shared data instruments for Research Electronic Data Capture (REDCap). J Biomed Inform 2013;46:259-65. 